\title{
A 900MHz RF Energy Harvesting Module
}

\author{
TARIS Thierry, VIGNERAS Valérie \\ IMS lab, University of Bordeaux \\ 33405 Talence Cedex, France
}

\author{
FADEL Ludivine \\ LAAS, University of Toulouse \\ 31000 Toulouse, France
}

\begin{abstract}
This paper presents a guideline to design and optimize a RF energy harvester operating in ISM Band at $902 \mathrm{MHz}$. The circuit is implemented on a standard FR4 board with commercially available off-the-shelf devices. The topology of the impedance transformation block is selected to reduce the losses which improves the overall performances of the system. The characterization of the harvesting module shows sensitivity of $-22.5 \mathrm{dBm}$ for a dc output voltage of $200 \mathrm{mV}$ up to $-11 \mathrm{dBm}$ for $1.08 \mathrm{~V}$. A wireless power transmission in an indoor environment is measured with a radiated source power of $16.8 \mathrm{dBm}$. The harvester exhibits a DC rectified voltage of $1.25 \mathrm{~V}$ at 0.5 meter and still $500 \mathrm{mV}$ at 1.5 meter.
\end{abstract}

\section{INTRODUCTION}

The finite life of electrical battery is encouraging the companies and researchers to come up with new ideas and technologies to extend the lifetime of mobile devices. For wireless miniature electronic the answer is available in capturing and storing the energy from external ambient sources, a technology known as energy harvesting. Different types of source are considered among them are: wind, solar, vibration, temperature gradient, thermoelectric, RadioFrequency (RF) and acoustic [1], [2], [3]. Among the top most power constrained applications are the Wireless Sensor Networks (WSN). Their developments, hardly driven by low cost, low power and compact implementations extensively investigate the energy scavenging. The huge deployment of RF communications over the three last decades makes RF energy available at "anytime and everywhere". It is a decisive asset to address power saving and energy management challenges in WSN. Furthermore the requirement for low cost solutions increases the interest for RF harvesting since it is supported by microelectronic market. Hence an important issue today is the development of inexpensive, compact and efficient RF energy scavengers for WSN applications.

This paper focuses on the design of a RF harvesting module operating in the $902-928 \mathrm{MHz}$ ISM Band. The demonstrator is exclusively developed with Commercially available Off-The-Shelf (COTS) devices to estimate the performances of a low cost solution. The design procedure of each featuring block is first detailed. The characterization of the module is then reported and compared to simulation results. The measurement of an indoor wireless power transmission (WPT) is presented and discussed. A figure of merit is introduced to compare the performances of the RF harvester with the state of art

\section{BUILDING BLOCK DESIGN AND CHARACTERISTICS}

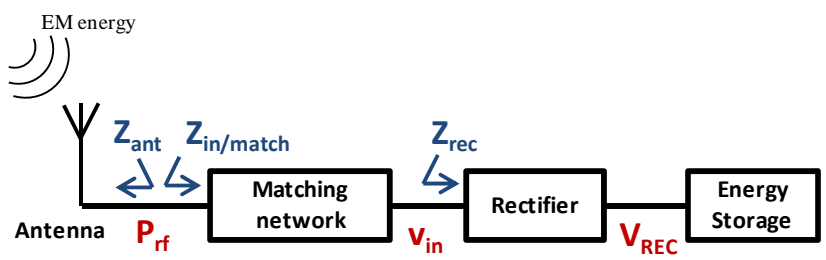

Figure 1. Building blocks of the RF harvesting module

The basic architecture of an RF energy harvester is presented in Fig. 1. The antenna performs the transduction of an electromagnetic energy into an electrical signal. To transfer a maximum of the signal to the rectifier a matching network is introduced. The rectifier converts the AC signal into a DC voltage charging a storage capacitor.

\section{A. Antenna}

An antenna is specified by its directivity, $G_{a n t}$, and its impedance, $Z_{\text {ant }}$. The selected element is here a quarter wave length dipole (Pulse Electronics) providing a $3 \mathrm{dBi}$ maximum gain in vertical position in a $60^{\circ}$ to $120^{\circ}$ aperture.

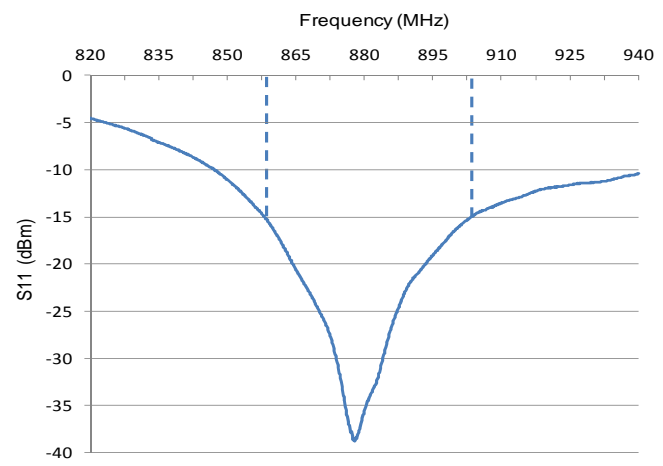

Figure 2. Return loss, $\mathrm{S}_{11}$, of the dipole antenna

The antenna impedance is characterized by its return loss $\mathrm{S}_{11}$, Fig. 2, measured with a HP 8720 network analyzer. Assuming a proper $50 \Omega$ matching for $\mathrm{S}_{11}$ below $-15 \mathrm{~dB}$, the antenna covers a 856 to $903 \mathrm{MHz}$ band, with a best matching at $878 \mathrm{MHz}$. 


\section{B. Rectifier}

Since the level of collected power, $\mathrm{P}_{\mathrm{rf}}$, is low, the AC to DC converter is based on a voltage multiplier to deliver an acceptable output DC voltage. A Cockcroft-Walton Voltage Doubler [4], Fig. 3(a), composes the elementary stage of the rectifier where $C_{1}(100 \mathrm{pF})$ and $\mathrm{D}_{1}$ form the negative clamp, $C_{2}(470 \mu \mathrm{F})$ and $D_{2}$ achieve peak rectification. $C_{2}$, smoothing the variations of $\mathrm{V}_{\mathrm{REC}}$, also acts as storage capacitor.

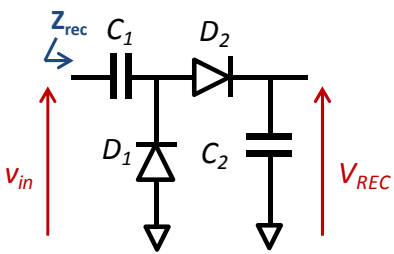

(a)

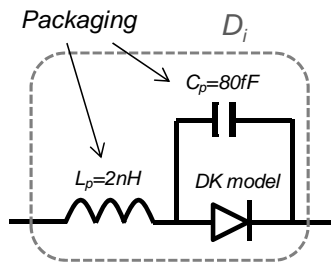

(b)
Figure 3. Schematic of a Greinacher Voltage Doubler (a) small signal model of a HSMS2852 zero bias diodes in a SOT323 packaging (b)

The diode skills are critical for the overall performances of the module. Schottky diode, offering low forward voltage, $\mathrm{V}_{\mathrm{F}}$, and high switching speed, $\mathrm{C}_{\mathrm{T}}$, are well suited for $\mathrm{RF}$ energy harvesting. The Avago HSMS-2852 Series featuring a $\mathrm{V}_{\mathrm{F}}$ of $150 \mathrm{mV}$, and a $\mathrm{C}_{\mathrm{T}}$ of $300 \mathrm{fF}$, has been selected. To improve the simulation accuracy, the diode model provided by the Design Kit (DK model) has been modified to take into account the packaging parasitic. It includes $\mathrm{L}_{\mathrm{P}}, 2 \mathrm{nH}$, and $\mathrm{C}_{\mathrm{P}}$, $80 \mathrm{fF}$ as reported in Fig.3 (b). The circuit of Fig.3 (a) is simulated in Advanced Design System (ADS) with Large Signal S-Parameter method. Device accesses and interconnections are modeled by tapers and microstrip lines, respectively. The real and imaginary parts of the input rectifier impedance, $\mathrm{Z}_{\mathrm{rec}}$, are depicted in Fig. 4 for an input power of $-20 \mathrm{dBm}$.

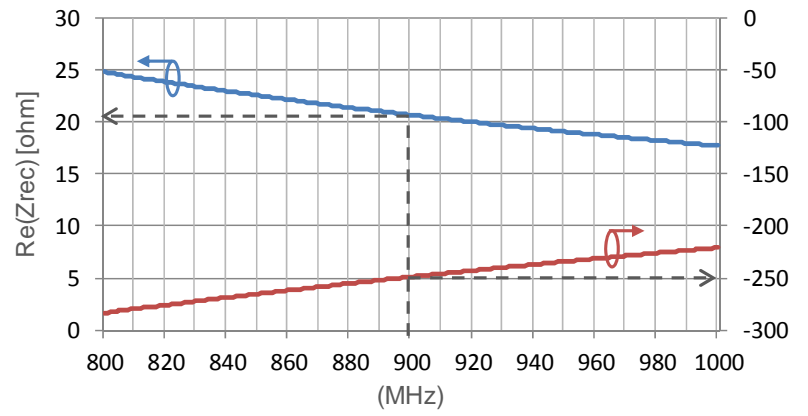

Figure 4. Simulated input impedance, $\mathrm{Z}_{\mathrm{rec}}$, of the single stage voltage doubler.

The quality factor, $\mathrm{Q}_{\mathrm{rf}}$, exploited by the matching network to boost the voltage at the input of the rectifier, is defined in (1). At $900 \mathrm{MHz}$, it varies from 7 to 11 with the input power.

$$
\mathrm{Q}_{\mathrm{rf}}=\frac{\left|\operatorname{Im}\left(\mathrm{Z}_{\text {rec }}\right)\right|}{\left|\operatorname{Re}\left(\mathrm{Z}_{\text {rec }}\right)\right|}
$$

\section{Matching Network}

A maximum of the available power, $\mathrm{P}_{\mathrm{rf}}$, is transferred from the antenna to the rectifier when an impedance equals to the conjugate of $\mathrm{Z}_{\mathrm{ant}}$, noted $\mathrm{Z}_{\mathrm{ant}}{ }^{*}$, is presented at the operating frequency. This principle is known as power matching.

Since $\mathrm{Z}_{\text {ant }} *$ and $\mathrm{Z}_{\text {rec}}$, reported in the smith chart of Fig. 5, do not complete these conditions, a matching network is inserted to transform the rectifier impedance to $\mathrm{Z}_{\mathrm{ant}}{ }^{*}$. To lower the losses and so improve voltage boosting, a solution embedding a reduced number of components is suited. The step by step design of the matching network is illustrated in Fig. 5. It combines a stub, a capacitor and a transmission line (microstrip type).

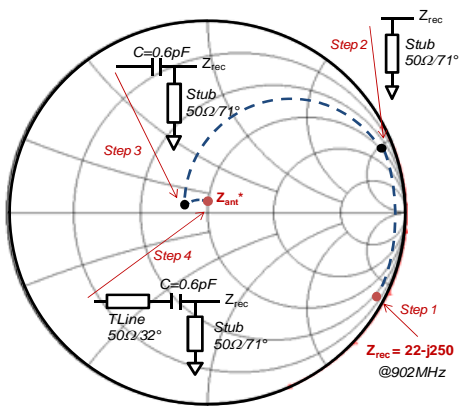

Figure 5. Constructive diagram of the matching network

\section{CARCATERISATION OF THE RF ENERGY HARVESTER}

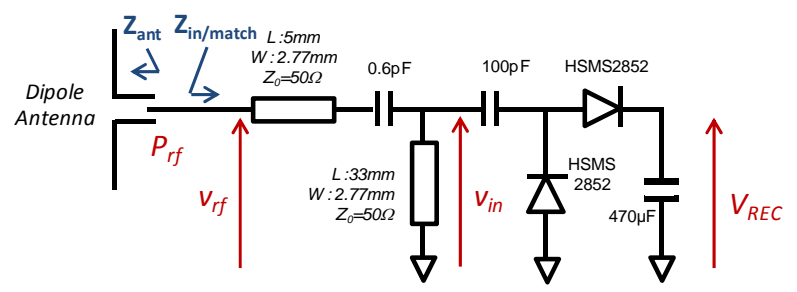

Figure 6. Schematic of the RF Energy harvesting module

The complete schematic of the harvester is presented in Fig. 6. The return loss $S_{11}$ at $Z_{\text {in/match }}$, input of the matching network/rectifier combination, is reported in Fig. 7 for an input power, $\mathrm{P}_{\mathrm{rf}}$, of $-20 \mathrm{dBm}$. In good agreement with simulation results, the minimum of measured $\mathrm{S}_{11},-31 \mathrm{~dB}$, yields at $902 \mathrm{MHz}$. This frequency, covered by the antenna bandwidth depicted in Fig. 2(b), will be further considered as the operating frequency of the system.

$$
\text { frequency }(\mathrm{MHz})
$$

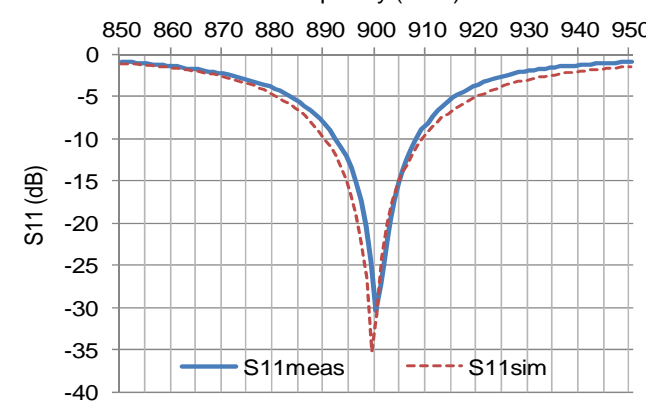

Figure 7. The return loss $S_{11}$ at $Z_{\text {in/match with }} P_{r f}=-20 d B m$ 
The return loss is also measured for various input power $\mathrm{P}_{\mathrm{rf}}$, from -25 to $-10 \mathrm{dBm}$, Fig. 8. The minimum of $\mathrm{S}_{11}$ shifts from 905 to $898 \mathrm{MHz}$ over the power range because of the non linear behavior of the diodes. Covered by the antenna bandwidth these variations do not alter the system characterization

frequency $(\mathrm{MHz})$

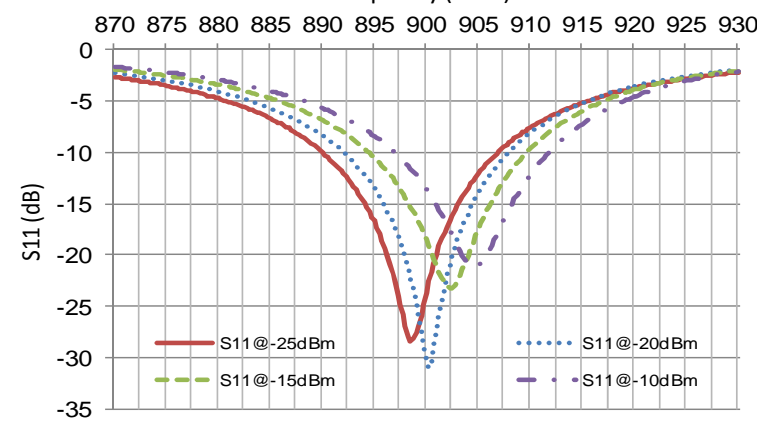

Figure 8. Measured $S_{11}$ at $Z_{\text {in/match }}$ for various $P_{\text {rf }}$

The rectified output voltage, $\mathrm{V}_{\mathrm{REC}}$, is derived in expression (2) based on [6] :

$$
\mathrm{V}_{\text {RECestim }}=2\left[\sqrt{2 \cdot R \mathrm{Re}\left[\mathrm{Z}_{\mathrm{rec}}\right] \cdot \mathrm{P}_{\mathrm{rf}} \cdot\left(1+\mathrm{Q}_{\mathrm{rf}}{ }^{2}\right)}-\mathrm{V}_{\mathrm{F}}\right]
$$

$\mathrm{Q}_{\mathrm{rf}}$ is the quality factor of the input network and $\mathrm{V}_{\mathrm{F}}$ is the diode threshold voltage defined in section II. To estimate $\mathrm{V}_{\mathrm{RECestim}}, \operatorname{Re}\left[\mathrm{Z}_{\mathrm{rec}}\right]$ and $\mathrm{Q}_{\mathrm{rf}}$ are extracted from Fig. 4. It is compared with the ADS simulations and measurement results in Fig. 9. $V_{\text {RECestim }}$ exhibits $+/-20 \%$ error with measurement results from -21 to $-13.5 \mathrm{~dB}$ with a best fit occurring at $P_{\mathrm{rf}}=-18 \mathrm{dBm}$. We can notice the close form of the simulated $\left(\mathrm{V}_{\mathrm{REC} \text { sim }}\right)$ and measured $\left(\mathrm{V}_{\mathrm{RECmeas}}\right)$ results over the entire power range.

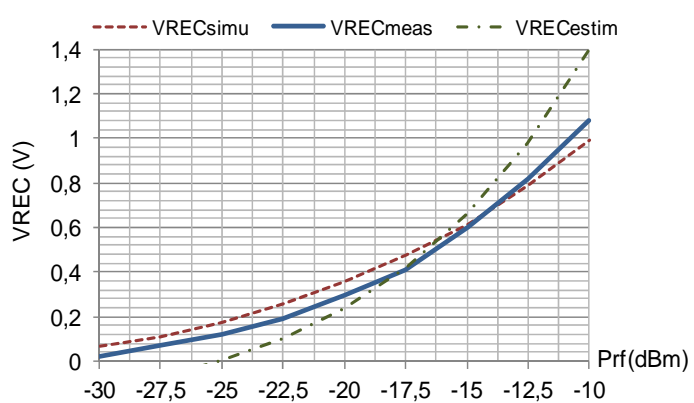

Figure 9. Rectified output voltage $\mathrm{V}_{\mathrm{REC}}$ versus input power $\mathrm{P}_{\mathrm{rf}}$ at $902 \mathrm{MHz}$

\section{Wireless POWER TRANSMISSION MEASUREMENTS}

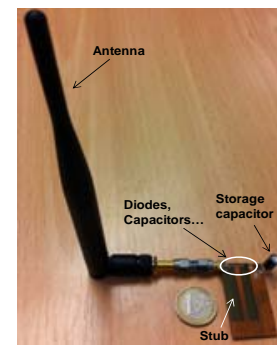

(a)

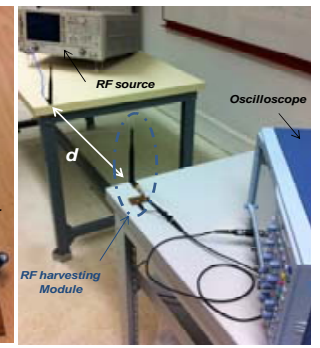

(b)
Figure 10. Single stage RF harvesting module (a) indoor WPT scene (b)
A picture of the RF energy harvester is depicted in Fig. 10 (a). The Printed Circuit Board (PCB) integrates the matching network, the rectifier and the storage capacitor on a standard $1.6 \mathrm{~mm}$ FR4 support. It is two time larger than a 1 euro coin. The antenna, in vertical position, is $16.5 \mathrm{~cm}$. The indoor measurements of wireless power transmission have been performed in a testing room of the lab, Fig. 10 (b).

A schematic of the WPT scene of Fig. 10(b) is proposed in Fig. 11. The RF source is a HP 8720 network analyzer connected to a dipole antenna. The RF harvester is the one presented in Fig. 10 (a). The voltage delivered by the harvester is measured with a Lecroy Wave pro 960 oscilloscope.

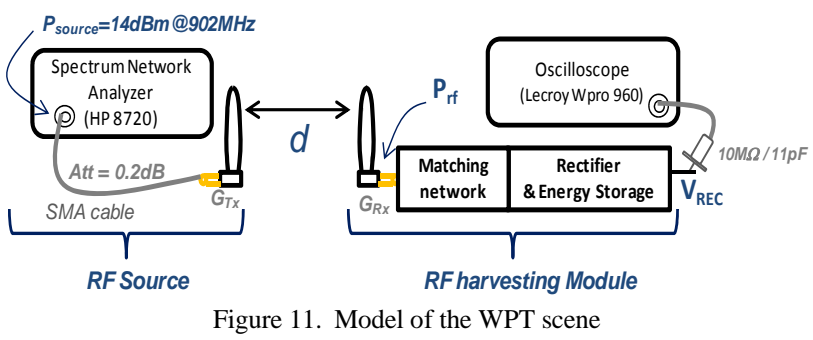

The relation between the power delivered by the source, $\mathrm{P}_{\text {source, }}$ and the available power, $\mathrm{P}_{\mathrm{rf}}$, at the $\mathrm{RF}$ harvester antenna is:

$$
P_{\text {rf }}=P_{\text {source }} \cdot G_{\text {ant } / T x} \cdot A(d) \cdot G_{\text {ant } / R x}
$$

With $A(d)$ the attenuation induced by the propagation of the signal in the air. We can find a lot of radio propagation model in the literature. Most of them are based on the free space path loss described by Friis [5] and reported in expression (4).

$$
\mathrm{A}(\mathrm{d})=\left(\frac{\lambda}{4 \pi \cdot \mathrm{d}}\right)^{2}
$$

With $\lambda$ the signal wave length and $d$ the distance between the source and the RF harvester in Fig.10 (b) \&11. Combining the expression (2), (3) and (4) we can estimate the rectified voltage $\mathrm{V}_{\mathrm{REC} \text { alc }}$ at a distance $d$ according (5):

$$
V_{R E \text { Calc }}=2\left[G_{\text {ant }} \frac{\lambda}{4 \pi d} \sqrt{2 . \Re e\left[Z_{r e c}\right] \cdot P_{r f} \cdot\left(1+Q_{r f}{ }^{2}\right)}-V_{F}\right]
$$

The network analyzer is in Continuous Wave (CW) mode, fixed at $902 \mathrm{MHz}$. The maximum power, $\mathrm{P}_{\text {source }}$, is $14 \mathrm{dBm}$. The overall radiated power is estimated to $16.8 \mathrm{dBm}$ including the cable loss, $0.2 \mathrm{~dB}$, and the antenna gain, $3 \mathrm{dBi}$. Fig. 12 represents the rectified voltage, $\mathrm{V}_{\mathrm{REC}}$, with the distance. $\mathrm{V}_{\text {RECcalc }}$ is calculed with (5). Likewise $\mathrm{V}_{\text {RECestim }}$ in Fig. 9, the calculated $\mathrm{V}_{\mathrm{REC} \text { calc }}$, proposed in (5), overestimates the rectified voltage for a couple of ten centimeters, which corresponds to a large harvested power, and underestimates it above 2 meters, for very low harvested power. The ADS simulations, performed with Ptolemy environment, and measurement results are very close with less than $15 \%$ error above 0.5 meter in Fig. 12. The harvester provides more than $500 \mathrm{mV}$ up to 1.5 meter. 


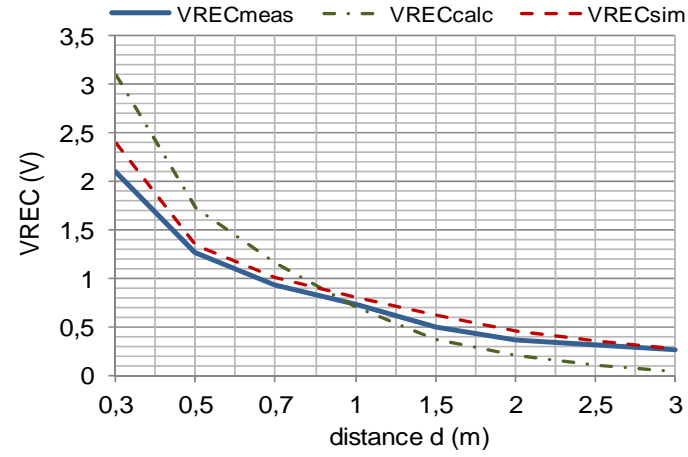

Figure 12; $\mathrm{V}_{\mathrm{REC}}$ versus distance $\mathrm{d}$ in WPT scene at $902 \mathrm{MHz}$

The regulation authorizes a maximum of $4 \mathrm{~W} / 36 \mathrm{dBm}$ EIRP in the $902-928 \mathrm{MHz}$ ISM Band. The $19 \mathrm{~dB}$ margin kept from this maximum is here imposed by the equipment - i.e. the network analyzer -. Some ADS simulations figure out the range of $500 \mathrm{mV}$ rectified voltage is extended to 15 meter under EIRP $_{\text {max }}$ conditions.

Multiple values of capacitor, type radial, were tested for the energy storage: from $10 \mu \mathrm{F}$ to $470 \mu \mathrm{F}$. No variation has been observed for the rectified voltage except the rising time needed to charge the capacitor at a fixed distance. Table I presents the measured rising time for $3.3 \mu \mathrm{F}, 10 \mu \mathrm{F}, 220 \mu \mathrm{F}$ and $470 \mu \mathrm{F}$. Assuming a typical RC charge, the output resistance of the rectifier, $R_{o u / r e c}$, is estimated based on expression (6):

$$
\mathrm{R}_{\text {out } / \text { rec }}=\frac{0.67 . \mathrm{t}_{\text {rise }}}{\mathrm{C}}
$$

TABLE I. Rising time and output resistance of the rectifier for various storage capacitors

\begin{tabular}{|c|c|c|c|c|}
\hline Capacitor $(\boldsymbol{\mu F})$ & 10 & 100 & 220 & 470 \\
\hline $\mathbf{t}_{\text {rise }}(\mathbf{s})$ & $0.73 \pm 0.03$ & $7.77 \pm 0.4$ & $16.7 \pm 1.5$ & $36 \pm 1.5$ \\
\hline $\mathbf{R}_{\text {out } / \mathbf{r e c}}(\mathbf{k} \Omega)$ & 50.2 & 52.1 & 50.8 & 51.3 \\
\hline
\end{tabular}

Some realizations of the state of art are reported in Table II. To discuss the performances of these RF energy harvesters we propose a figure of merit, expression (7), rating the voltage efficiency per stage.

$$
F O M=\frac{V_{\text {REC }}[\mathrm{V}] . \text { frequency }[\mathrm{GHz}]}{\mathrm{P}_{\mathrm{rf}}[\mathrm{mW}] \cdot \mathrm{N}[\text { number of stage }]}
$$

Passive devices, and so input matching networks, exhibit a low quality factor in CMOS technologies because of the low resistivity of the substrate. In the meantime the threshold voltage of MOS based diodes, ranging from 250 to $350 \mathrm{mV}$ in sub $100 \mathrm{~nm}$ processes, is high compared to the forward voltage of COTS schottky devices, typical $150 \mathrm{mV}$ for a HSMS2852. As matter of consequences COTS harvesters exhibit a better FOM [this work], [9] than their IC counterparts [6], [7], [8].

This analysis figures out System On a Chip (SOC) implementations pushed by the development of CMOS technologies limit the performances in the context of RF energy harvesting. Focusing on efficiency and cost issues COTS solutions are better suited.

TABLE II. Comparison with the state of art

\begin{tabular}{|c|c|c|c|c|c|}
\hline Reference & $\begin{array}{c}\text { This } \\
\text { work }\end{array}$ & {$[6]$} & {$[7]$} & {$[8]$} & {$[9]$} \\
\hline Freq (GHz) & 0.9 & 0.92 & 0.9 & 0.91 & 2.4 \\
\hline P $_{\text {rf }}(\mathbf{d B m})$ & -10 & -10 & -22 & -15 & -10 \\
\hline $\mathbf{V}_{\text {rec }}(\mathbf{V})$ & 1.08 & 3.6 & 1 & 2.8 & 0.6 \\
\hline $\begin{array}{c}\text { Number of } \\
\text { stage }\end{array}$ & 1 & 4 & 17 & 16 & 1 \\
\hline Technology & $\begin{array}{c}\text { HSMS2 } \\
852\end{array}$ & $\begin{array}{c}\text { CMOS } \\
0.18 \mu \mathrm{m}\end{array}$ & $\begin{array}{c}\text { CMOS } \\
90 \mathrm{~nm}\end{array}$ & $\begin{array}{c}\text { CMOS } \\
250 \mathrm{~nm}\end{array}$ & $\begin{array}{c}\text { HSMS } \\
2850\end{array}$ \\
\hline FOM & 9.7 & 8.3 & 8.8 & 5.3 & 14.4 \\
\hline
\end{tabular}

V. CONCLUSION

A low cost RF energy harvesting module has been designed and implemented on a FR4 board with COTS devices. The design procedure including parasitic elements is detailed. It is validated by the close form between the simulated and measured data of the RF harvester. A figure of merit rating the voltage efficiency per stage is proposed. The circuit developed in this work exhibits a larger FOM than CMOS counterparts which confirms the interest in developing RF energy harvester with COTS. Illuminated by a RF source radiating a power of $16.8 \mathrm{dBm}$ in an indoor environment, the harvesting module provides a rectified DC voltage of $1.25 \mathrm{~V}$ at 0.5 meter and $500 \mathrm{mV}$ at 1.5 meter over a $470 \mu \mathrm{F}$ storage capacitor.

\section{REFERENCES}

[1] J.A. Paradiso and T. Starner, "Energy scavenging for mobile and wireless electronics,” IEEE Pervasive Computing, Vol. 4, Issue: 1, pp. 18- 27, Jan-March 2005.

[2] A. Chandrakasan, R. Amirtharajah, S. Cho, J. Goodman, G. Konduri, J. Kulik, W. Rabiner and A. Wang, "Design considerations for distributed microsensor systems," Proceedings of the IEEE Custom Integrated Circuits, pp. 279-286, San Diego, USA, 1999.

[3] Y. Hu, M. Sawan and M. N. El-Gamal, “An Integrated Power Recovery Module Dedicated to Implantable Electronic Devices,” Analog Integrated Circuits and Signal Processing, Vol. 43, No. 2, pp. 171-181, May 2005.

[4] J. D. Cockcroft, E. T. S. Walton, "Further developments on the method of obtaining high-velocity positive ions,” in Proc. Roy. Soc., London, U.K., 1932, p. 131-619.

[5] H.T. Friis, "A Note on a Simple Transmission Formula," Proc. IRE, vol. 34, no. 5, pp. 254-256, May 1946.

[6] Shameli, A., Safarian, A., Rofougaran, A., Rofougaran, M. \& De Flaviis, F., "Power Harvester Design for Passive UHF RFID Tag Using a Voltage Boosting Technique", Microwave Theory and Techniques, IEEE Transactionson, vol. 55; 55, no. 6, pp. 1089-1097, 2007.

[7] Papotto, G., Carrara, F., Palmisano, G., "A 90-nm CMOS ThresholdCompensated RF Energy Harvester", Solid-State Circuits, IEEE Journal of, On page(s): 1985 - 1997, Volume: 46 Issue: 9, Sept. 2011

[8] T. Le, K. Mayaram, and T. Fiez, "Efficient far-field radio frequency energy harvesting for passively powered sensor networks,” IEEE J. Solid-State Circuits, vol. 43, no. 5, pp. 1287-1302, May 2008.

[9] A.S. Boaventura et al, "Maximizing DC power in energy harvesting circuits using multisine excitation " IEEE Microwave Symposium Digest, CDROM, Baltimore, USA, June 2011. 